\title{
On the Characterization of Some Classes of Four-Dimensional Matrices and Almost $B$-Summable Double Sequences
}

\author{
Orhan Tug \\ Department of Mathematics Education, Ishik University, Ishik Campus, 100 Meter Street, Erbil, Iraq \\ Correspondence should be addressed to Orhan Tug; orhan.tug@ishik.edu.iq
}

Received 13 January 2018; Accepted 12 March 2018; Published 19 April 2018

Academic Editor: Alfred Peris

Copyright (C) 2018 Orhan Tug. This is an open access article distributed under the Creative Commons Attribution License, which permits unrestricted use, distribution, and reproduction in any medium, provided the original work is properly cited.

We firstly summarize the related literature about $B(r, s, t, u)$-summability of double sequence spaces and almost $B(r, s, t, u)$ summable double sequence spaces. Then we characterize some new matrix classes of $\left(\mathscr{L}_{s^{\prime}}: \mathscr{C}_{f}\right),\left(B\left(\mathscr{L}_{s^{\prime}}\right): \mathscr{C}_{f}\right)$, and $\left(\mathscr{L}_{s^{\prime}}: B\left(\mathscr{C}_{f}\right)\right)$ of four-dimensional matrices in both cases of $0<s^{\prime} \leq 1$ and $1<s^{\prime}<\infty$, and we complete this work with some significant results.

\section{Preliminaries, Background, and Notations}

We denote the set of all complex valued double sequence by $\Omega$ which is a vector space with coordinatewise addition and scalar multiplication. Any subspace of $\Omega$ is called a double sequence space. A double sequence $x=\left(x_{m n}\right)$ of complex numbers is called bounded if $\|x\|_{\infty}=\sup _{m, n \in \mathbb{N}}\left|x_{m n}\right|<\infty$, where $\mathbb{N}=\{0,1,2, \ldots\}$. The space of all bounded double sequences is denoted by $\mathscr{M}_{u}$ which is a Banach space with the norm $\|\cdot\|_{\infty}$. Consider the double sequence $x=\left(x_{m n}\right) \in \Omega$. If for every $\epsilon>0$ there exists a natural number $n_{0}=n_{0}(\epsilon)$ and $l \in \mathbb{C}$ such that $\left|x_{m n}-l\right|<\epsilon$ for all $m, n>n_{0}$, then the double sequence $x$ is said to be convergent in Pringsheim's sense to the limit point $l$; say that $p-\lim _{m, n \rightarrow \infty} x_{m n}=l$, where $\mathbb{C}$ indicates the complex field. The space $\mathscr{C}_{p}$ denotes the set of all convergent double sequences in Pringsheim's sense. Although every convergent single sequence is bounded, this is not hold for double sequences in general. That is, there are such double sequences which are convergent in Pringsheim's sense but not bounded. Actually, Boos [1, p. 16] defined the sequence $x=\left(x_{m n}\right)$ by

$$
x_{m n}= \begin{cases}n, & m=0, n \in \mathbb{N} ; \\ 0, & m \geq 1, n \in \mathbb{N} .\end{cases}
$$

Then it is clearly seen that $p-\lim _{m, n \rightarrow \infty} x_{m n}=0$ but $\|x\|_{\infty}=$ $\sup _{m, n \in \mathbb{N}}\left|x_{m n}\right|=\infty$, so $x \in \mathscr{C}_{p}-\mathscr{M}_{u}$. The set $\mathscr{C}_{b p}$ denotes the space of both bounded and convergent double sequences; that is, $\mathscr{C}_{b p}=\mathscr{C}_{p} \cap \mathscr{M}_{u}$. Hardy [2] showed that a double sequence $x=\left(x_{m n}\right)$ is said to converge regularly to $l$ if $x \in \mathscr{C}_{p}$ and the limits $x_{m}:=\lim _{n} x_{m n},(m \in \mathbb{N})$ and $x_{n}:=\lim _{m} x_{m n},(n \in \mathbb{N})$ exist, and the $\operatorname{limits}_{\lim } \lim _{n} x_{m n}$ and $\lim _{n} \lim _{m} x_{m n}$ exist and are equal to the $p$-limit of $x$. Moreover, by $\mathscr{C}_{b p 0}$ and $\mathscr{C}_{r 0}$, we may denote the spaces of all null double sequences contained in the sequence spaces $\mathscr{C}_{b p}$ and $\mathscr{C}_{r}$, respectively. Móricz [3] proved that the double sequence spaces $\mathscr{C}_{b p}, \mathscr{C}_{b p 0}, \mathscr{C}_{r}$, and $\mathscr{C}_{r 0}$ are Banach spaces with the norm $\|\cdot\|_{\infty}$. The space $\mathscr{L}_{q}$ of all absolutely $q$-summable double sequences corresponding to the space $\ell_{q}$ of $q$-summable single sequences was defined by Başar and Sever [4]; that is,

$$
\mathscr{L}_{q}:=\left\{x=\left(x_{k l}\right) \in \Omega: \sum_{k, l}\left|x_{k l}\right|^{q}<\infty\right\} \text {, }
$$

$$
(1 \leq q<\infty)
$$

which is a Banach space with the norm $\|\cdot\|_{q}$. Then, the space $\mathscr{L}_{u}$ which is a special case of the space $\mathscr{L}_{q}$ with $q=1$ is introduced by Zeltser [5].

Let $\lambda$ be a double sequence space and converging with respect to some linear convergence rule is $\vartheta-\lim : \lambda \rightarrow \mathbb{C}$. Then, the sum of a double series $\sum_{i, j} x_{i j}$ relating to this rule is defined by $\vartheta-\sum_{i, j} x_{i j}=\vartheta-\lim _{m, n \rightarrow \infty} \sum_{i, j=0}^{m, n} x_{i j}$. Throughout the paper, the summations from 0 to $\infty$ without limits, that is, $\sum_{i, j} x_{i j}$, mean that $\sum_{i, j=0}^{\infty} x_{i j}$.

Here and after, unless otherwise stated, we consider that $\vartheta$ denotes any of the symbols $p, b p$, or $r$. 
The $\alpha$-dual $\lambda^{\alpha}$, the $\beta(\vartheta)-$ dual $\lambda^{\beta(9)}$ with respect to the $\vartheta$-convergence, and the $\gamma$-dual $\lambda^{\gamma}$ of double sequence space $\lambda$ are, respectively, defined by

$$
\begin{aligned}
\lambda^{\alpha} & :=\left\{a=\left(a_{k l}\right) \in \Omega: \sum_{k, l}\left|a_{k l} x_{k l}\right|<\infty \quad \forall x=\left(x_{k l}\right)\right. \\
& \in \lambda\}, \\
\lambda^{\beta(\vartheta)} & :=\left\{a=\left(a_{k l}\right) \in \Omega: \vartheta-\sum_{k, l} a_{k l} x_{k l} \quad \text { exists } \forall x\right. \\
& \left.=\left(x_{k l}\right) \in \lambda\right\}, \\
\lambda^{\gamma} & :=\left\{a=\left(a_{k l}\right) \in \Omega: \sup _{m, n \in \mathbb{N}}\left|\sum_{k, l=0}^{m, n} a_{k l} x_{k l}\right|<\infty \quad \forall x\right. \\
& \left.=\left(x_{k l}\right) \in \lambda\right\} .
\end{aligned}
$$

It is easy to see for any two spaces $\lambda$ and $\mu$ of double sequences that $\mu^{\alpha} \subset \lambda^{\alpha}$ whenever $\lambda \subset \mu$ and $\lambda^{\alpha} \subset \lambda^{\gamma}$. Additionally, it is known that the inclusion $\lambda^{\alpha} \subset \lambda^{\beta(\vartheta)}$ holds while the inclusion $\lambda^{\beta(9)} \subset \lambda^{\gamma}$ does not hold, since the 9 -convergence of the double sequence of partial sum of a double series does not guarantee its boundedness.

Here, we shall be concerned with four-dimensional matrix transformation from any double sequence space $\lambda$ to any double sequence space $\mu$. Given any four-dimensional infinite matrix $A=\left(a_{m n k l}\right)$, where $m, n, k, l \in \mathbb{N}$, any double sequence $x=\left(x_{k l}\right)$, we write $A x=\left\{(A x)_{m n}\right\}_{m, n \in \mathbb{N}}$, the $A$-transform of $x$, exists for every sequence $x=\left(x_{k l}\right) \in \lambda$ and it is in $\mu$, where

$$
(A x)_{m n}=\vartheta-\sum_{k, l} a_{m n k l} x_{k l} \quad \text { for each } m, n \in \mathbb{N} .
$$

The four-dimensional matrix domain has fundamental importance for this article. Therefore, this concept is presented in this paragraph. The 9 -summability domain $\lambda_{A}^{(9)}$ of $A$ in a space $\lambda$ of double sequences is described as

$$
\begin{aligned}
\lambda_{A}^{(\vartheta)} & =\left\{x=\left(x_{k l}\right) \in \Omega: A x\right. \\
& \left.=\left(\vartheta-\sum_{k, l} a_{m n k l} x_{k l}\right)_{m, n \in \mathbb{N}} \text { exists and is in } \lambda\right\} .
\end{aligned}
$$

Notation (4) says that $A$ maps the space $\lambda$ into the space $\mu$ if $\lambda \subset \mu_{A}^{(9)}$ and we denote the set of all four-dimensional matrices, transforming the space $\lambda$ into the space $\mu$, by $(\lambda: \mu)$. Thus, $A=\left(a_{\text {mnkl }}\right) \in(\lambda: \mu)$ if and only if the double series on the right side of (4) converges in the sense of $\vartheta$ for each $m, n \in$
$\mathbb{N}$; that is, $A_{m n} \in \lambda^{\beta(9)}$ for all $m, n \in \mathbb{N}$ and we have $A x \in \mu$ for all $x \in \lambda$, where $A_{m n}=\left(a_{m n k l}\right)_{k, l \in \mathbb{N}}$ for all $m, n \in \mathbb{N}$. Moreover, the following definitions are significant in order to classify the four-dimensional matrices. A four-dimensional matrix $A$ is called $\mathscr{C}_{\vartheta}-$ conservative if $\mathscr{C}_{\vartheta} \subset\left(\mathscr{C}_{\vartheta}\right)_{A}$ and is called $\mathscr{C}_{\vartheta}$ - regular if it is $\mathscr{C}_{\vartheta}-$ conservative and

$$
\begin{array}{r}
\vartheta-\lim A x=\vartheta-\lim _{m, n \rightarrow \infty}(A x)_{m n}=\vartheta-\lim _{m, n \rightarrow \infty} x_{m n}, \\
\text { where } x=\left(x_{m n}\right) \in \mathscr{C}_{\vartheta} .
\end{array}
$$

One can be obtained by using the notations of Zeltser [6] that we may define the double sequences $e^{k l}=\left(e_{m n}^{k l}\right), e^{1}, e_{k}$, and $e$ by

$$
e_{m n}^{k l}= \begin{cases}1, & (k, l)=(m, n) ; \\ 0, & \text { otherwise. }\end{cases}
$$

$e^{1}=\sum_{k} e^{k l}, e_{k}=\sum_{l} e^{k l}$, and $e=\sum_{k l} e^{k l}$ for all $k, l, m, n \in \mathbb{N}$ and we may write the set $\Phi$ by $\Phi=\operatorname{span}\left\{e^{k l}: k, l \in \mathbb{N}\right\}$.

In order to establish a new sequence space, special triangular matrices were previously used. These new spaces derived by the domain of matrices are expansion or the contraction of the original space, in general. Adams [7] defined that the four-dimensional infinite matrix $A=\left(a_{m n k l}\right)$ is called a triangular matrix if $a_{m n k l}=0$ for $k>m$ or $l>n$ or both. We also say by [7] that an infinite matrix $A=\left(a_{m n k l}\right)$ is said to be a triangular if $a_{m n m n} \neq 0$ for all $m, n \in \mathbb{N}$. Moreover, Cooke [8] showed that every triangular matrix has a unique inverse which is also a triangular matrix.

The four-dimensional generalized difference matrix $B(r, s, t, u)=\left\{b_{m n k l}(r, s, t, u)\right\}$ and matrix domain of it on some double sequence spaces were recently defined by Tuğ and Başar [9] and studied by Tuğ [10-12]. The matrix $B(r, s, t, u)=\left\{b_{m n k l}(r, s, t, u)\right\}$ was defined by Tuğ and Başar [9] as

$$
b_{m n k l}(r, s, t, u):= \begin{cases}s u, & (k, l)=(m-1, n-1) \\ s t, & (k, l)=(m-1, n) \\ r u, & (k, l)=(m, n-1) \\ r t, & (k, l)=(m, n) \\ 0, & \text { otherwise }\end{cases}
$$

for $r, s, t, u \in \mathbb{R} \backslash\{0\}$ and for all $m, n, k, l \in \mathbb{N}$. Therefore, the $B(r, s, t, u)$-transform of a double sequence $x=\left(x_{m n}\right)$ was defined by

$$
\begin{aligned}
y_{m n} & :=\{B(r, s, t, u) x\}_{m n}=\sum_{k, l} b_{m n k l}(r, s, t, u) x_{k l} \\
& =s u x_{m-1, n-1}+s t x_{m-1, n}+r u x_{m, n-1}+r t x_{m n}
\end{aligned}
$$


for all $m, n \in \mathbb{N}$. Moreover, the matrix $B^{-1}(r, s, t, u)=F(r, s$, $t, u)=\left\{f_{\text {mnkl }}(r, s, t, u)\right\}$ which is the inverse of the matrix $B(r, s, t, u)$ was calculated as

$$
\begin{aligned}
& f_{\text {mnkl }}(r, s, t, u) \\
& \quad:= \begin{cases}\frac{(-s / r)^{m-k}(-u / t)^{n-l}}{r t}, & 0 \leq k \leq m, 0 \leq l \leq n, \\
0, & \text { otherwise }\end{cases}
\end{aligned}
$$

for all $m, n, k, l \in \mathbb{N}$. Furthermore, Tuğ and Başar [9] obtained the relation between $x=\left(x_{m n}\right)$ and $y=\left(y_{m n}\right)$ by

$$
x_{m n}=\frac{1}{r t} \sum_{k, l=0}^{m, n}\left(\frac{-s}{r}\right)^{m-k}\left(\frac{-u}{t}\right)^{n-l} y_{k l} \quad \forall m, n \in \mathbb{N} .
$$

Tŭg and Başar [9] and Tuğ [10] have introduced and studied the new double sequence spaces $B\left(\mathscr{M}_{u}\right), B\left(\mathscr{C}_{p}\right)$, $B\left(\mathscr{C}_{b p}\right), B\left(\mathscr{C}_{r}\right)$, and $B\left(\mathscr{L}_{q}\right)$ as the domain of four-dimensional generalized difference matrix $B(r, s, t, u)$ in the spaces $\mathscr{M}_{u}$, $\mathscr{C}_{p}, \mathscr{C}_{b p}, \mathscr{C}_{r}$, and $\mathscr{L}_{q}$, respectively. That is,

$$
\begin{aligned}
& B\left(\mathscr{M}_{u}\right):=\left\{x=\left(x_{m n}\right) \in \Omega: \sup _{m, n \in \mathbb{N}}\left|\{B(r, s, t, u) x\}_{m n}\right|\right. \\
& <\infty\}, \\
& B\left(\mathscr{C}_{p}\right):=\left\{x=\left(x_{m n}\right) \in \Omega: \exists l \in \mathbb{C} \ni p\right. \\
& \left.\quad-\lim _{m, n \rightarrow \infty}\left|\{B(r, s, t, u) x\}_{m n}-l\right|=0\right\}, \\
& B\left(\mathscr{C}_{b p}\right):=\left\{x=\left(x_{m n}\right) \in \Omega: B(r, s, t, u) x \in \mathscr{C}_{b p}\right\}, \\
& B\left(\mathscr{C}_{r}\right):=\left\{x=\left(x_{m n}\right) \in \Omega: B(r, s, t, u) x \in \mathscr{C}_{r}\right\}, \\
& B\left(\mathscr{L}_{q}\right):=\left\{x=\left(x_{m n}\right) \in \Omega: \sum_{m, n}\left|\{B(r, s, t, u) x\}_{m n}\right|^{q}\right. \\
& \quad<\infty\}, \quad 0<q<\infty .
\end{aligned}
$$

Lorentz [13] introduced the concept of almost convergence for single sequence and Moricz and Rhoades [14] extended and studied this concept for double sequence. A double sequence $x=\left(x_{k l}\right)$ of complex numbers is said to be almost convergent to a generalized limit $L$ if

$$
p-\lim _{q, q^{\prime} \rightarrow \infty} \sup _{m, n>0}\left|\frac{1}{(q+1)\left(q^{\prime}+1\right)} \sum_{k=m}^{m+q} \sum_{l=n}^{n+q^{\prime}} x_{k l}-L\right|=0 .
$$

In this case, $L$ is called the $f_{2}$-limit of the double sequence $x$. Throughout the paper, $\mathscr{C}_{f}$ denotes the space of all almost convergent double sequences; that is,

$$
\begin{aligned}
& \mathscr{C}_{f}:=\left\{x=\left(x_{k l}\right) \in \Omega: \exists L \in \mathbb{C} \ni p\right. \\
&- \lim _{q, q^{\prime} \rightarrow \infty} \sup _{m, n>0}\left|\frac{1}{(q+1)\left(q^{\prime}+1\right)} \sum_{k=m}^{m+q} \sum_{l=n}^{n+q^{\prime}} x_{k l}-L\right| \\
&=0, \quad \text { uniformly in } m, n\} .
\end{aligned}
$$

It is known that a convergent double sequence need not be almost convergent. But it is well known that every bounded convergent double sequence is also almost convergent and every almost convergent double sequence is bounded. That is, the inclusion $\mathscr{C}_{b p} \subset \mathscr{C}_{f} \subset \mathscr{M}_{u}$ holds, and each inclusion is proper. A double sequence $x=\left(x_{k l}\right)$ is called almost Cauchy which was introduced by Čunjalo [15] if for every $\epsilon>0$ there exists a positive integer $K$ such that

$$
\begin{aligned}
& \mid \frac{1}{\left(q_{1}+1\right)\left(q_{1}^{\prime}+1\right)} \sum_{k=m_{1}}^{m_{1}+q_{1}} \sum_{l=n_{1}}^{n_{1}+q_{1}^{\prime}} x_{k l} \\
& -\frac{1}{\left(q_{2}+1\right)\left(q_{2}^{\prime}+1\right)} \sum_{k=m_{2}}^{m_{2}+q_{2}} \sum_{l=n_{2}}^{n_{2}+q_{2}^{\prime}} x_{k l} \mid<\epsilon
\end{aligned}
$$

for all $q_{1}, q_{1}^{\prime}, q_{2}, q_{2}^{\prime}>K$ and $\left(m_{1}, n_{1}\right),\left(m_{2}, n_{2}\right) \in \mathbb{N} \times \mathbb{N}$. Mursaleen and Mohiuddine [16] proved that every double sequence is almost convergent if and only if it is almost Cauchy.

Moricz and Rhoades [14] considered that four-dimensional matrices transforming every almost convergent double sequence into a $b p$-convergent double sequence with the same limit. Almost conservative and almost regular matrices for single sequences were characterized by King [17] and almost $\mathscr{C}_{\vartheta}$-conservative and almost $\mathscr{C}_{\vartheta}$-regular fourdimensional matrices for double sequences were defined and characterized by Zeltser et al. [18]. Mursaleen [19] introduced the almost strongly regularity for double sequences. A fourdimensional matrix $A=\left(a_{m n k l}\right)$ is called almost strongly regular if it transforms every almost convergent double sequence into an almost convergent double sequence with the same limit.

Definition 1 (see [18]). A four-dimensional matrix $A=$ $\left(a_{\text {mnkl }}\right)$ is said to be almost $\mathscr{C}_{\vartheta}$-conservative matrix if it transforms every 9 -convergent double sequence $x=\left(x_{k l}\right)$ into an almost convergent double sequence space; that is, $A=\left(a_{m n k l}\right) \in\left(\mathscr{C}_{\vartheta}: \mathscr{C}_{f}\right)$.

Definition 2 (see [18]). A four-dimensional matrix $A=$ $\left(a_{\text {mnkl }}\right.$ ) is said to be almost $\mathscr{C}_{\vartheta}$-regular if it is $\mathscr{C}_{\vartheta}$-conservative and $f_{2}-\lim A x=\vartheta-\lim x$ for all $x \in \mathscr{C}_{\vartheta}$. 
Tuğ $[11,12]$ has recently studied new almost convergent double sequence spaces $B\left(\mathscr{C}_{f}\right)$ and $B\left(\mathscr{C}_{f_{0}}\right)$ as the domain of four-dimensional generalized difference matrix $B(r, s, t, u)$ in the spaces $\mathscr{C}_{f}$ and $\mathscr{C}_{f_{0}}$, respectively. That is,

$$
\begin{aligned}
& B\left(\mathscr{C}_{f}\right):=\left\{x=\left(x_{k l}\right) \in \Omega: \exists L \in \mathbb{C} \ni p\right. \\
& -\lim _{q, q^{\prime} \rightarrow \infty} \sup _{m, n>0}\left|\frac{1}{(q+1)\left(q^{\prime}+1\right)} \sum_{k=m}^{m+q} \sum_{l=n}^{n+q^{\prime}}(B x)_{k l}-L\right| \\
& =0, \quad \text { uniformly in } m, n\}, \\
& B\left(\mathscr{C}_{f_{0}}\right):=\left\{x=\left(x_{k l}\right) \in \Omega: p\right. \\
& \quad-\lim _{q, q^{\prime} \rightarrow \infty} \sup _{m, n>0}\left|\frac{1}{(q+1)\left(q^{\prime}+1\right)} \sum_{k=m}^{m+q} \sum_{l=n}^{n+q^{\prime}}(B x)_{k l}\right| \\
& =0, \quad \text { uniformly in } m, n\} .
\end{aligned}
$$

In this paper, as natural continuation of $[10,11]$, we characterize some new matrix classes $\left(\mathscr{L}_{s^{\prime}}: \mathscr{C}_{f}\right),\left(B\left(\mathscr{L}_{s^{\prime}}\right)\right.$ : $\left.\mathscr{C}_{f}\right)$, and $\left(\mathscr{L}_{s^{\prime}}: B\left(\mathscr{C}_{f}\right)\right)$ of four-dimensional matrices in both cases $0<s^{\prime}<1$ and $1<s^{\prime}<\infty$. Throughout the paper, we suppose that the terms of double sequence $x=\left(x_{m n}\right)$ and $y=\left(y_{m n}\right)$ are connected with relation (11) and the four-dimensional generalized difference matrix $B(r, s, t, u)=$ $\left(b_{m n k l}(r, s, t, u)\right)$ will be presented with $B=\left(b_{m n k l}\right)$.

\section{Main Results}

In this section, we characterize some new four-dimensional matrix classes $\left(\mathscr{L}_{s^{\prime}}: \mathscr{C}_{f}\right),\left(B\left(\mathscr{L}_{s^{\prime}}\right): \mathscr{C}_{f}\right),\left(\mathscr{L}_{s^{\prime}}: B\left(\mathscr{C}_{f}\right)\right)$ in both cases of $0<s \leq 1$ and $1<s<\infty$, and $\left(B\left(\mathscr{C}_{f}\right): \mathscr{M}_{u}\right)$. Then we complete this section with some significant results of four-dimensional matrix mapping via the dual summability methods for double sequences which has been introduced and studied by Başar [20] and Yeşilkayagil and Başar [21] and has been recently applied in $[10,12]$.

Theorem 3. Let $A=\left(a_{m n k l}\right)$ be a four-dimensional infinite matrix. Then the following statements hold. only if

(a) Let $0<s^{\prime} \leq 1$. Then, $A=\left(a_{m n k l}\right) \in\left(\mathscr{L}_{s^{\prime}}: \mathscr{C}_{f}\right)$ if and

$$
\begin{aligned}
& \sup _{m, n, k, l \in \mathbb{N}}\left|a_{m n k l}\right|<\infty, \\
& \quad \exists\left(a_{k l}\right) \in \mathbb{C} \text { such that } f_{2}-\lim _{m, n \rightarrow \infty} a_{m n k l}=a_{k l}
\end{aligned}
$$

(b) Let $1<s^{\prime}<\infty$. Then, $A=\left(a_{m n k l}\right) \in\left(\mathscr{L}_{s^{\prime}}: \mathscr{C}_{f}\right)$ if and only if condition (18) holds and

$$
\sup _{m, n \in \mathbb{N}} \sum_{k, l}\left|a_{m n k l}\right|^{s^{\prime}}<\infty .
$$

Proof. (a) Let $0<s^{\prime} \leq 1$ and $A=\left(a_{\text {mnkl }}\right) \in\left(\mathscr{L}_{s^{\prime}}: \mathscr{C}_{f}\right)$. Then, $A x$ exists and is in $\mathscr{C}_{f}$ for all $x=\left(x_{k l}\right) \in \mathscr{L}_{s^{\prime}}$. Since the inclusion $\left(\mathscr{L}_{s^{\prime}}: \mathscr{C}_{f}\right) \subset\left(\mathscr{L}_{s^{\prime}}: \mathscr{M}_{u}\right)$ holds, then we may say that condition (17) is necessary. Moreover, since $A x$ exists and is in $\mathscr{C}_{f}$ for all $x=\left(x_{k l}\right) \in \mathscr{L}_{s^{\prime}}$, it is also provided for $x=e^{k l} \in \mathscr{L}_{s^{\prime}}$ that $A e^{k l}=\left(a_{m n k l}\right)_{m, n \in \mathbb{N}} \in \mathscr{C}_{f}$ which gives us condition (18) which is also necessary.

Conversely, suppose that conditions (17) and (18) hold and $x=\left(x_{k l}\right)$ be any double sequence in $\mathscr{L}_{s^{\prime}}$. Since $A_{m n} \in$ $\mathscr{L}_{s^{\prime}}^{\beta(9)}$ by corollary 3.2 of [22] for each $m, n \in \mathbb{N}, A x$ exists. By using conditions (17) and (18) for each $k, l \in \mathbb{N}$, we have the following inequality:

$$
\left|a_{k l}\right|=f_{2}-\lim _{m, n \rightarrow \infty}\left|a_{m n k l}\right| \leq \sup _{m, n \in \mathbb{N}}\left|a_{m n k l}\right|
$$

then we may say that $a_{k l} \in \mathscr{M}_{u}$. Thus, the series $\sum_{k, l} a_{k l} x_{k l}$ is convergent for all $x \in \mathscr{L}_{s^{\prime}}$. Moreover, by condition (18) we may say that for every $\epsilon>0$ there exists a positive $n_{0} \in \mathbb{N}$ such that

$$
\frac{1}{(q+1)\left(q^{\prime}+1\right)} \sum_{k=j}^{j+q} \sum_{l=i}^{i+q^{\prime}}\left|a_{m n k l}-a_{k l}\right|<\epsilon
$$

for all $m, n>n_{0}$. Thus

$$
\begin{aligned}
& \frac{1}{(q+1)\left(q^{\prime}+1\right)} \\
& \cdot \sum_{k=j}^{j+q} \sum_{l=i}^{i+q^{\prime}}\left(\left|\sum_{v, v^{\prime}}^{k, l} a_{m n v, v^{\prime}} x_{v, v^{\prime}}-\sum_{v, v^{\prime}}^{k, l} a_{v, v^{\prime}} x_{v, v^{\prime}}\right|^{s^{\prime}}\right) \\
& =\frac{1}{(q+1)\left(q^{\prime}+1\right)} \\
& \cdot \sum_{k=j}^{j+q} \sum_{l=i}^{i+q^{\prime}}\left(\left|\sum_{v, v^{\prime}}^{k, l}\left(a_{m n v, v^{\prime}}-a_{v, v^{\prime}}\right) x_{v, v^{\prime}}\right|^{s^{\prime}}\right) \\
& \leq \frac{\epsilon^{s^{\prime}}}{(q+1)\left(q^{\prime}+1\right)} \sum_{k=j}^{j+q} \sum_{l=i}^{i+q^{\prime}}\left(\sum_{v, v^{\prime}}^{k, l}\left|x_{v, v^{\prime}}\right|\right) \\
& <\frac{\epsilon^{s^{\prime}}}{(q+1)\left(q^{\prime}+1\right)} \sum_{k=j}^{j+q} \sum_{l=i}^{i+q^{\prime}}\left(\sum_{v, v^{\prime}}^{k, l}\left|x_{v, v^{\prime}}\right|^{s^{\prime}}\right) .
\end{aligned}
$$

It gives us that $p-\lim _{q, q^{\prime} \rightarrow \infty}\left(1 /(q+1)\left(q^{\prime}+1\right)\right) \sum_{k=j}^{j+q} \sum_{l=i}^{i+q^{\prime}}(A x)_{k l}$ exists; that is, $A x \in \mathscr{C}_{f}$.

This completes the proof of $(a)$.

(b) Let $1<s^{\prime}<\infty$. The necessity of conditions (18) and (19) may be easily shown in the similar method which is used in (a) with the Hölder inequality. So we pass the repetition. 
Conversely, suppose that conditions (18) and (19) hold and $x=\left(x_{k l}\right)$ be a double sequence in the space $\mathscr{L}_{s^{\prime}}$. It can be written with (18) for all $k, l \in \mathbb{N}$ that

$$
\begin{aligned}
\sum_{v, v^{\prime}=0}^{k, l}\left|a_{v, v^{\prime}}\right|^{s^{\prime}} & =f_{2}-\lim _{m, n \rightarrow \infty} \sum_{v, v^{\prime}=0}^{k, l}\left|a_{m n v, v^{\prime}}\right|^{s^{\prime}} \\
& \leq \sup _{m, n \in \mathbb{N}_{v, v^{\prime}=0}} \sum_{m n v, v^{\prime}}^{k, l} \mid a^{s^{\prime}}<\infty ;
\end{aligned}
$$

say that $a=\left(a_{v, v^{\prime}}\right) \in \mathscr{L}_{s^{\prime}}$. Therefore, the double series $\sum_{v, v^{\prime}=0}^{k, l} a_{v, v^{\prime}} x_{v, v^{\prime}}$ is convergent for every $x \in \mathscr{L}_{s^{\prime}}$. Moreover, for any given $\epsilon>0$, there exist $N_{0} \in \mathbb{N}$ such that

$$
\begin{aligned}
& \frac{1}{(q+1)\left(q^{\prime}+1\right)} \sum_{k=j}^{j+q} \sum_{l=i}^{i+q^{\prime}}\left(\sum_{v, v^{\prime}=0}^{k, l}\left|\left(a_{m n v, v^{\prime}}-a_{v, v^{\prime}}\right)\right|^{s^{\prime}}\right) \\
& <\epsilon
\end{aligned}
$$

for every $m, n>N_{0}$. So, by using Hölder's inequality with relations (23) and (24) we have power to write that

$$
\begin{aligned}
& \frac{1}{(q+1)\left(q^{\prime}+1\right)} \\
& \cdot \sum_{k=j}^{j+q} \sum_{l=i}^{i+q^{\prime}}\left|\sum_{v, v^{\prime}=0}^{k, l} a_{m n v, v^{\prime}} x_{v, v^{\prime}}-\sum_{v, v^{\prime}=0}^{k, l} a_{v, v^{\prime}} x_{v, v^{\prime}}\right| \\
& =\frac{1}{(q+1)\left(q^{\prime}+1\right)} \\
& \cdot \sum_{k=j}^{j+q} \sum_{l=i}^{i+q^{\prime}}\left|\sum_{v, v^{\prime}=0}^{k, l}\left(a_{m n v, v^{\prime}}-a_{v, v^{\prime}}\right) x_{v, v^{\prime}}\right|
\end{aligned}
$$

for all $m, n \in \mathbb{N}$. After passing $p$-limit as $q, q^{\prime} \rightarrow \infty$ we can say that $A x \in \mathscr{C}_{f}$. This last step completes the proof.

Theorem 4. Let $A=\left(a_{m n k l}\right)$ be a four-dimensional infinite matrix. Then the following statements hold.

(a) Let $0<s^{\prime} \leq 1$. Then, $A=\left(a_{\text {mnkl }}\right) \in\left(\mathscr{L}_{s^{\prime}}: B\left(\mathscr{C}_{f}\right)\right)$ if and only if

$$
\begin{aligned}
\sup _{m, n, k, l \in \mathbb{N}}\left|\sum_{i, j=0}^{m, n} b_{m n i j} a_{i j k l}\right| & <\infty, \\
\exists e_{k l} & \in \Omega \ni f_{2}-\lim _{m, n \rightarrow \infty} \sum_{i, j=0}^{m, n} b_{m n i j} a_{i j k l} \\
& =e_{k l} \quad \forall k, l \in \mathbb{N} .
\end{aligned}
$$

(b) $1<s^{\prime}<\infty$. Then, $A=\left(a_{m n k l}\right) \in\left(\mathscr{L}_{s^{\prime}}: B\left(\mathscr{C}_{f}\right)\right)$ if and only if condition (27) holds and

$$
\sup _{m, n \in \mathbb{N}} \sum_{k, l}\left|\sum_{i, j=0}^{m, n} b_{m n i j} a_{i j k l}\right|^{s^{\prime}}<\infty
$$

Proof. Suppose that the four-dimensional matrix $A=$ $\left(a_{m n k l}\right) \in\left(\mathscr{L}_{s^{\prime}}: B\left(\mathscr{C}_{f}\right)\right)$. Then, $A x$ exist and is in $B\left(\mathscr{C}_{f}\right)$ for all $x \in \mathscr{L}_{s^{\prime}}$. Since $A x \in B\left(\mathscr{C}_{f}\right)$ then we can say that $B(A x) \in \mathscr{C}_{f}$. Moreover, we may have the following equality:

$$
\sum_{i, j=0}^{m, n} b_{m n i j} \sum_{v, v^{\prime}=0}^{k, l} a_{i j v, v^{\prime}} x_{v, v^{\prime}}=\sum_{v, v^{\prime}=0}^{k, l}\left(\sum_{i, j=0}^{m, n} b_{m n i j} a_{i j v, v^{\prime}}\right) x_{v, v^{\prime}}
$$

holds for all $m, n, k, l \in \mathbb{N}$. Therefore, we have $B(A x)=(B A) x$ by taking limit as $k, l \rightarrow \infty$ in (29). Now we can define the new four-dimensional matrix $B A=G=\left(g_{m n k l}\right)$ as

$$
g_{m n k l}=\sum_{i, j=0}^{m, n} b_{m n i j} a_{i j v, v^{\prime}}
$$

So we can write here that $A x \in B\left(\mathscr{C}_{f}\right)$ whenever $x \in \mathscr{L}_{s^{\prime}}$ if and only if $G x \in \mathscr{C}_{f}$ whenever $x \in \mathscr{L}_{s^{\prime}}$ which says that $G=\left(g_{\text {mnkl }}\right) \in\left(\mathscr{L}_{s^{\prime}}: \mathscr{C}_{f}\right)$. Thus conditions (17) and (18) hold for $0<s^{\prime} \leq 1$, and conditions (18) and (19) hold for $1<s^{\prime}<\infty$ with $g_{m n k l}$ instead of $a_{m n k l}$. This gives us the results as desired.

Theorem 5. Let $A=\left(a_{m n k l}\right)$ be a four-dimensional infinite matrix. Then the following statements hold.

(a) Let $0<s^{\prime} \leq 1$. Then, $A=\left(a_{m n k l}\right) \in\left(B\left(\mathscr{L}_{s^{\prime}}\right): \mathscr{C}_{f}\right)$ if and only if

$$
\begin{aligned}
& \sup _{m, n, k, l \in \mathbb{N}}\left|\sum_{i, j=k, l}^{m, n}\left(\frac{-s}{r}\right)^{i-k}\left(\frac{-u}{t}\right)^{j-l} \frac{a_{m n i j}}{r t}\right|<\infty, \\
& \exists e_{k l} \in \Omega \ni f_{2}-\lim _{m, n \rightarrow \infty} \sum_{i, j=k, l}^{m, n}\left(\frac{-s}{r}\right)^{i-k}\left(\frac{-u}{t}\right)^{j-l} \frac{a_{m n i j}}{r t} \\
& =e_{k l} .
\end{aligned}
$$

(b) Let $1<s^{\prime}<\infty$. Then, $A=\left(a_{\text {mnkl }}\right) \in\left(B\left(\mathscr{L}_{s^{\prime}}\right): \mathscr{C}_{f}\right)$ if and only if condition (32) holds and

$$
\sup _{m, n \in \mathbb{N}} \sum_{k, l}\left|\sum_{i, j=k, l}^{m, n}\left(\frac{-s}{r}\right)^{i-k}\left(\frac{-u}{t}\right)^{j-l} \frac{a_{m n i j}}{r t}\right|^{s^{\prime}}<\infty .
$$

Proof. Suppose that the four-dimensional matrix $A=$ $\left(a_{m n k l}\right) \in\left(B\left(\mathscr{L}_{s^{\prime}}\right): \mathscr{C}_{f}\right)$. Then, as the method used in the proof of Theorem 4, $A x$ exist and is in $\mathscr{C}_{f}$ for all $x \in B\left(\mathscr{L}_{s^{\prime}}\right)$. Since $x \in B\left(\mathscr{L}_{s^{\prime}}\right)$ then we can say that $B x=y \in \mathscr{L}_{s^{\prime}}$ and the equality

$$
\begin{aligned}
& \sum_{i, j=0}^{m, n} a_{m n i j} x_{v, v^{\prime}} \\
& \quad=\sum_{v, v^{\prime}=0}^{k, l}\left(\sum_{i, j=k, l}^{m, n}\left(\frac{-s}{r}\right)^{i-k}\left(\frac{-u}{t}\right)^{j-l} \frac{a_{m n i j}}{r t}\right) y_{v, v^{\prime}}
\end{aligned}
$$

holds for all $m, n, k, l \in \mathbb{N}$. Therefore, we have $A x=A B^{-1} y=$ $E y$ by taking limit as $k, l \rightarrow \infty$ in (34). Now we may define the following four-dimensional matrix $A B^{-1}=E=\left(e_{m n k l}\right)$ as

$$
e_{m n k l}=\sum_{i, j=k, l}^{m, n}\left(\frac{-s}{r}\right)^{i-k}\left(\frac{-u}{t}\right)^{j-l} \frac{a_{m n i j}}{r t} .
$$


So we can write here that $A x \in \mathscr{C}_{f}$ whenever $x \in B\left(\mathscr{L}_{s^{\prime}}\right)$ if and only if $E y \in \mathscr{C}_{f}$ whenever $y \in \mathscr{L}_{s^{\prime}}$ which says that $E=$ $\left(e_{m n k l}\right) \in\left(\mathscr{L}_{s^{\prime}}: \mathscr{C}_{f}\right)$. Thus conditions (17) and (18) hold for $0<s^{\prime} \leq 1$, and conditions (18) and (19) hold for $1<s^{\prime}<\infty$ with $e_{m n k l}$ instead of $a_{m n k l}$. This gives us the expected results and completes the proof.

Corollary 6. Suppose that the elements of four-dimensional infinite matrices $A=\left(a_{m n k l}\right)$ and $H=\left(h_{m n k l}\right)$ are connected with relation

$$
h_{m n k l}=\sum_{i, j=k, l}^{m, n} b_{m n i j} e_{i j k l} \quad \forall m, n, k, l \in \mathbb{N} .
$$

Then the following statements hold.

(i) Let $0<s^{\prime} \leq 1$. Then $A \in\left(B\left(\mathscr{L}_{s^{\prime}}\right): B\left(\mathscr{C}_{f}\right)\right)$ iff (17) and (18) hold with $h_{m n k l}$ instead of $a_{m n k l}$.

(ii) Let $1<s^{\prime}<\infty$. Then $A \in\left(B\left(\mathscr{L}_{s^{\prime}}\right): B\left(\mathscr{C}_{f}\right)\right)$ iff (18) and (19) hold with $h_{m n k l}$ instead of $a_{m n k l}$.

\section{Conclusion}

The concept of almost convergence of single sequence was introduced by Lorentz [13]. In 2010, Mursaleen [23] investigated the certain properties of the space of almost convergent sequences denoted by $f$. Then many of the mathematicians have studied the matrix domain on almost null and almost convergent sequences spaces (see [24-27]).

The almost convergence for double sequence was introduced by Moricz and Rhoades [14] and studied on by many researchers (see [16, 28-36]). Yeşilkayagil and Basar [37] recently studied the topological properties of the spaces of almost null and almost convergent double sequences.

The domain of four-dimensional generalized difference matrix $B(r, s, t, u)$ on some double sequence spaces $B\left(\mathscr{C}_{p}\right)$, $B\left(\mathscr{C}_{b p}\right), B\left(\mathscr{C}_{r}\right)$, and $B\left(\mathscr{L}_{q}\right)$ has been introduced and studied by Tuğ and Başar [9] and by Tuğ [10]. They examined some topological properties. Furthermore, they determined the $\alpha-, \beta(\vartheta)-$, and $\gamma$-duals of some new double sequence spaces and characterized some classes of four-dimensional matrix transformations related to the new double sequence spaces.

The domain of the four-dimensional generalized difference matrix $B(r, s, t, u)$ in the spaces of almost null and almost convergent double sequences has been recently introduced and studied by Tuğ $[11,12]$. He examined some topological properties and determined $\alpha-, \beta(b p)-$, and $\gamma$-duals of the space $B\left(\mathscr{C}_{f}\right)$. Moreover, he characterized some new classes of four-dimensional matrix mappings related to the sequence space $B\left(\mathscr{C}_{f}\right)$.

In this work, we tried to fill one of the gaps in the fourdimensional matrix characterization of the matrix classes $\left(\mathscr{L}_{s^{\prime}}: \mathscr{C}_{f}\right),\left(B\left(\mathscr{L}_{s^{\prime}}\right): \mathscr{C}_{f}\right)$, and $\left(\mathscr{L}_{s^{\prime}}: B\left(\mathscr{C}_{f}\right)\right)$ in both cases of $0<s^{\prime} \leq 1$ and $1<s^{\prime}<\infty$. The characterization of the matrix classes $\left(\mathscr{C}_{f}: \mathscr{C}_{p}\right),\left(\mathscr{C}_{f}: \mathscr{C}_{r}\right),\left(B\left(\mathscr{C}_{f}\right): \mathscr{C}_{p}\right)$, and $\left(B\left(\mathscr{C}_{f}\right): \mathscr{C}_{r}\right)$ and calculating the $\beta(\theta)$ dual of the space $B\left(\mathscr{C}_{f}\right)$ where $\theta=(p, r)$ are still open problems for readers.

\section{Data Availability}

The data used to support the findings of this study are available from the corresponding author upon request.

\section{Conflicts of Interest}

The author declares here that there are no conflicts of interest.

\section{Authors' Contributions}

The author characterized some new four-dimensional matrix classes and proved the necessary and sufficient conditions. In the last section, some studies were summarized and some open problems were given by the author. The author read and approved the final manuscript.

\section{Acknowledgments}

The author would like to thank Professor Dr. Feyzi Başar for his careful reading and valuable suggestions on the first version of this paper which improved the presentation and readability. The author also would like thank to the audience of seminar sessions which was held by Mathematics Education Department of Ishik University for their valuable suggestions in order to improve the quality of this study. This work was supported by the Research Center of Ishik University, Erbil-IRAQ.

\section{References}

[1] J. Boos, Classical and Modern Methods in Summability, Oxford University Press, Oxford, UK, 2000.

[2] G. H. Hardy, "On the convergence of certain multiple series," Mathematical Proceedings of the Cambridge Philosophical Society, vol. 19, pp. 86-95, 1916-1919.

[3] F. Móricz, "Extensions of the spaces $\mathrm{c}$ and $\mathrm{c} 0$ from single to double sequence," Acta Mathematica Hungarica, vol. 57, no. 12, pp. 129-136, 1991.

[4] F. Başar and Y. Sever, "The space Lq of double sequences," Mathematical Journal of Okayama University, vol. 51, pp. 149$157,2009$.

[5] M. Zeltser, "On conservative matrix methods for double sequence spaces," Acta Mathematica Hungarica, vol. 95, no. 3, pp. 225-242, 2002.

[6] M. Zeltser, Investigation of Double Sequence Spaces by Soft and Hard Analytical Methods, vol. 25 of Dissertationes Mathematicae Universitatis Tartuensis, Tartu University Press, Tartu, Estonia, 2001.

[7] C. R. Adams, "On non-factorable transformations of double sequences," Proceedings of the National Acadamy of Sciences of the United States of America, vol. 19, no. 5, pp. 564-567, 1933.

[8] R. G. Cooke, Infinite Matrices and Sequence Spaces, Macmillan, London, UK, 1950.

[9] O. Tŭ̆ and F. Başar, "Four-dimensional generalized difference matrix and some double sequence spaces," in Proceedings of the 3rd International Conference on Analysis and Applied Mathematics, ICAAM 2016, vol. 1759, No. 1., AIP Publishing, September 2016. 
[10] O. Tuğ, "Four-dimensional generalized difference matrix and some double sequence spaces," Journal of Inequalities and Applications, vol. 1, no. 149, 22 pages, 2017.

[11] O. Tuğ, "Four-dimensional generalized difference matrix and almost convergent double sequence spaces," in Functional analysis in interdisciplinary applications, vol. 216 of Springer Proc. Math. Stat., pp. 83-87, Springer, Cham, Switzerland, 2017.

[12] O. Tug, "On almost B-summable double sequence spaces," Journal of Inequalities and Applications, vol. 1, no. 9, 19 pages, 2018.

[13] G. G. Lorentz, "A contribution to the theory of divergent sequences," Acta Mathematica, vol. 80, pp. 167-190, 1948.

[14] F. Moricz and B. E. Rhoades, "Almost convergence of double sequences and strong regularity of summability matrices," Mathematical Proceedings of the Cambridge Philosophical Society, vol. 104, no. 2, pp. 283-294, 1988.

[15] F. Čunjalo, "Almost convergence of double sequences-some analogies between measure and category," Mathematica Macedonica, vol. 5, pp. 21-24, 2007.

[16] M. Mursaleen and S. A. Mohiuddine, Convergence Methods for Double Sequences and Applications, Springer, New Delhi, India, 2014.

[17] J. P. King, "Almost summable sequences," Proceedings of the American Mathematical Society, vol. 17, pp. 1219-1225, 1966.

[18] M. Zeltser, M. Mursaleen, and S. A. Mohiuddine, "On almost conservative matrix methods for double sequence spaces," Publicationes Mathematicae, vol. 75, no. 3-4, pp. 387-399, 2009.

[19] M. Mursaleen, "Almost strongly regular matrices and a core theorem for double sequences," Journal of Mathematical Analysis and Applications, vol. 293, no. 2, pp. 523-531, 2004.

[20] F. Başar, Summability Theory and Its Applications, Bentham Science Publishers, Ltd., Oak Park, IL, USA, 2012.

[21] M. Yeşilkayagil and F. Başar, "Four dimensional dual and dual of the new sort summability methods," Contemporary Analysis and Applied Mathematics, vol. 3, no. 1, pp. 13-29, 2015.

[22] M. Yeşilkayagil and F. Başar, "On the domain of Riesz mean in the space Ls," Filomat, vol. 31, no. 4, pp. 925-940, 2017.

[23] M. Mursaleen, "Almost convergence and some related methods," in Modern Methods of Analysis and Its Applications, pp. 1-10, Anamaya Publishers, New Delhi, India, 2010.

[24] F. Başar and M. Kirişçi, "Almost convergence and generalized difference matrix," Computers and Mathematics with Applications, vol. 61, no. 3, pp. 602-611, 2011.

[25] O. Tuğ and F. Basar, "On the spaces of Nörlund almost null and Nörlund almost convergent sequences," Filomat, vol. 30, no. 3, pp. 773-783, 2016.

[26] K. Kayaduman and M. Şengönül, "The spaces of Cesàro almost convergent sequences and core theorems," Acta Mathematica Scientia B, vol. 32, no. 6, pp. 2265-2278, 2012.

[27] M. Şengönül and K. Kayaduman, "On the Riesz almost convergent sequences space," Abstract and Applied Analysis, vol. 2012, Article ID 691694, 18 pages, 2012.

[28] M. Mursaleen and E. Savas, "Almost regular matrices for double sequences," Studia Scientiarum Mathematicarum Hungarica, vol. 40, no. 1-2, pp. 205-212, 2003.

[29] F. Móricz and B. E. Rhoades, "Some characterizations of almost convergence for single and double sequences," in Proceedings of the 3rd Annual Meeting of the International Workshop in Analysis and its Applications, vol. 48(62) of Publ. Inst. Math.(Beograd)(NS)., pp. 61-68, 1990.
[30] S. A. Mohiuddine, "An application of almost convergence in approximation theorems," Applied Mathematics Letters, vol. 24, no. 11, pp. 1856-1860, 2011.

[31] M. Mursaleen and S. A. Mohiuddine, "Banach limit and some new spaces of double sequences," Turkish Journal of Mathematics, vol. 36, no. 1, pp. 121-130, 2012.

[32] S. A. Mohiuddine and A. Alotaibi, "Almost conservative fourdimensional matrices through de la Vallée-Poussin mean," Abstract and Applied Analysis, vol. 2014, Article ID 412974, 6 pages, 2014.

[33] M. Mursaleen and S. A. Mohiuddine, "Regularly $\sigma$-conservative and -coercive four dimensional matrices," Computers \& Mathematics with Applications, vol. 56, no. 6, pp. 1580-1586, 2008.

[34] S. A. Mohiuddine and A. Alotaibi, "Generalized almost convergence and core theorems of double sequences," Abstract and Applied Analysis, vol. 2014, Article ID 152910, 7 pages, 2014.

[35] M. Mursaleen and S. A. Mohiuddine, "Almost bounded variation of double sequences and some four dimensional summability matrices," Publicationes Mathematicae, vol. 75, no. 3-4, pp. 495-508, 2009.

[36] M. Mursaleen and S. A. Mohiuddine, "Some new double sequence spaces of invariant means," Glasnik Matematicki, vol. 45, no. 1, pp. 139-153, 2010.

[37] M. Yeșilkayagil and F. Basar, "Some topological properties of the spaces of almost null and almost convergent double sequences," Turkish Journal of Mathematics, vol. 40, no. 3, pp. 624-630, 2016. 


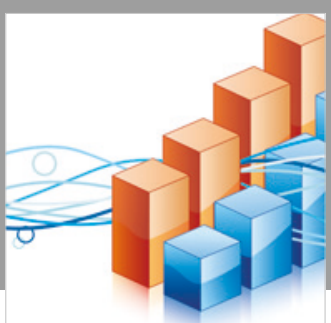

Advances in

Operations Research

\section{-n-m}
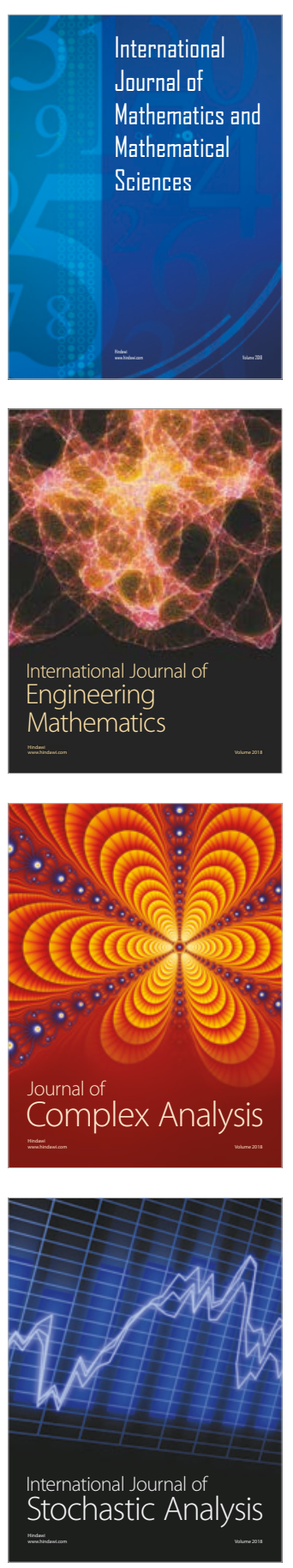
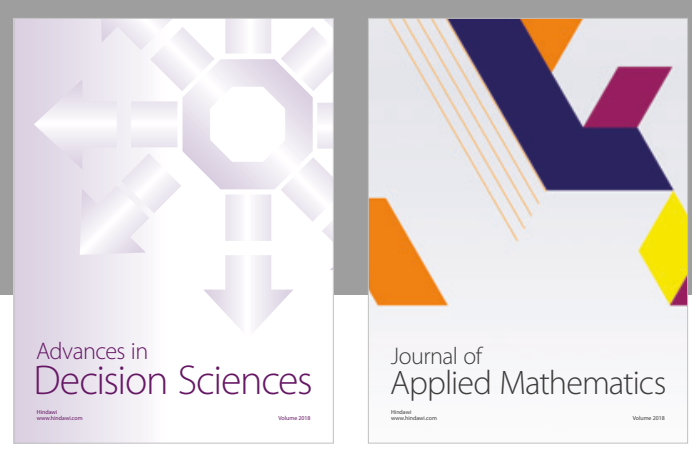

Journal of

Applied Mathematics
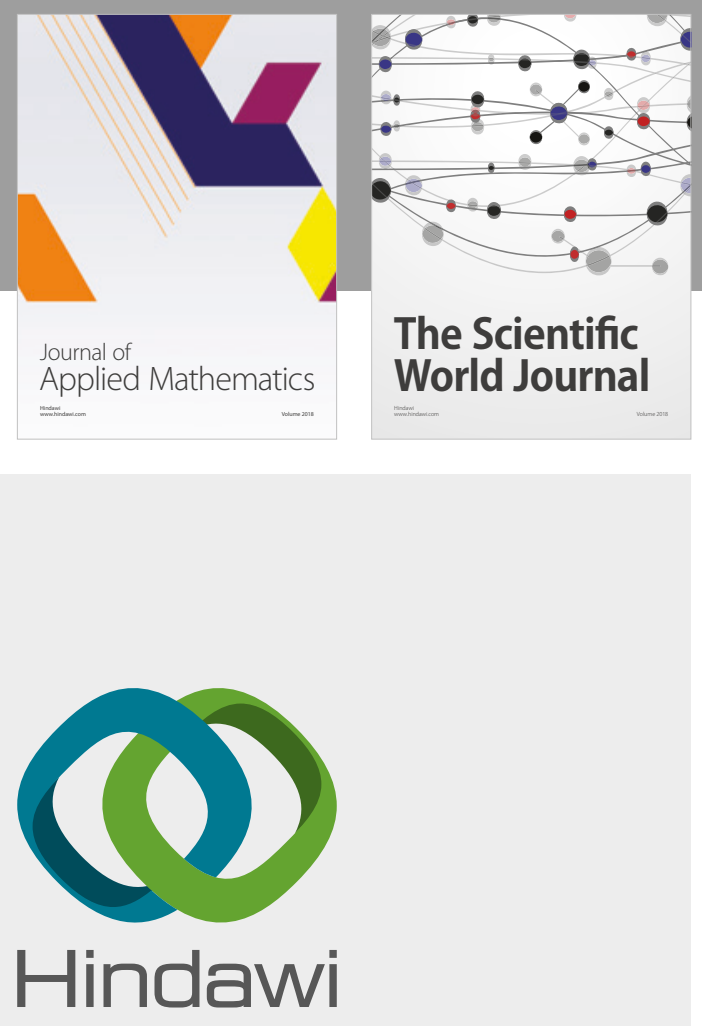

Submit your manuscripts at

www.hindawi.com

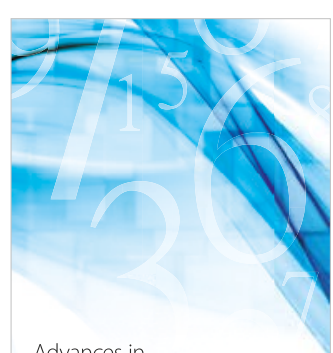

Advances in
Numerical Analysis
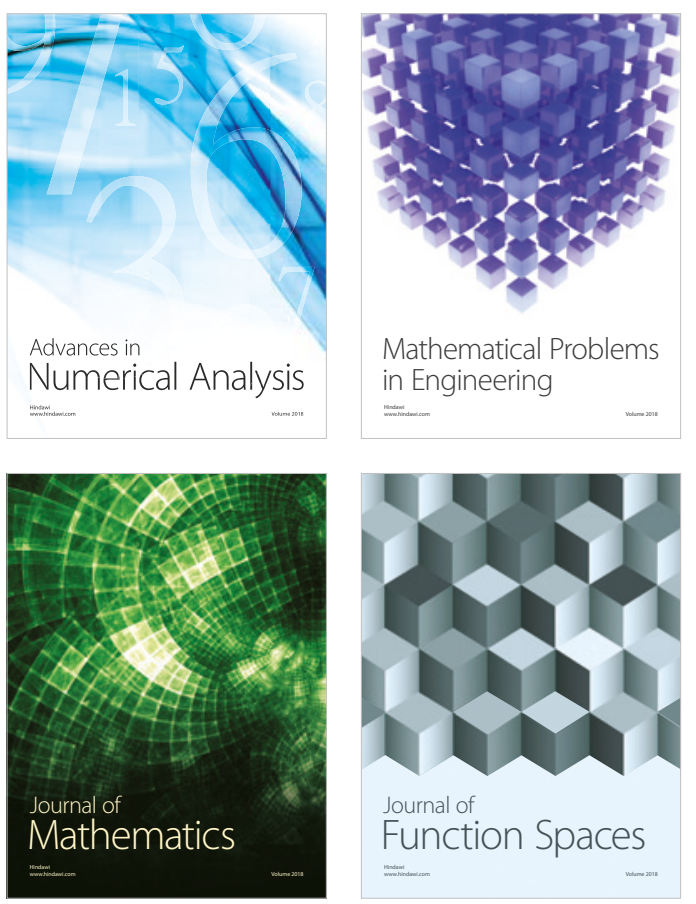

Mathematical Problems in Engineering

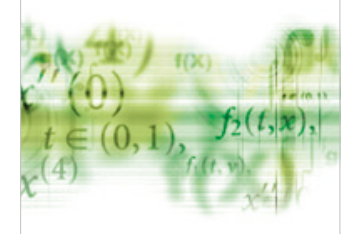

International Journal of

Differential Equations

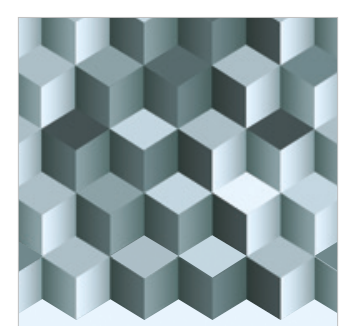

Journal of

Function Spaces

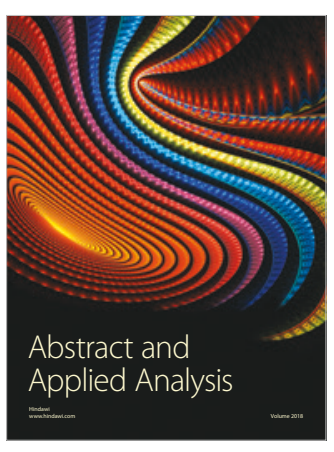

The Scientific

World Journal

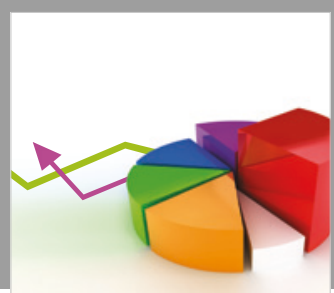

Journal of

Probability and Statistics
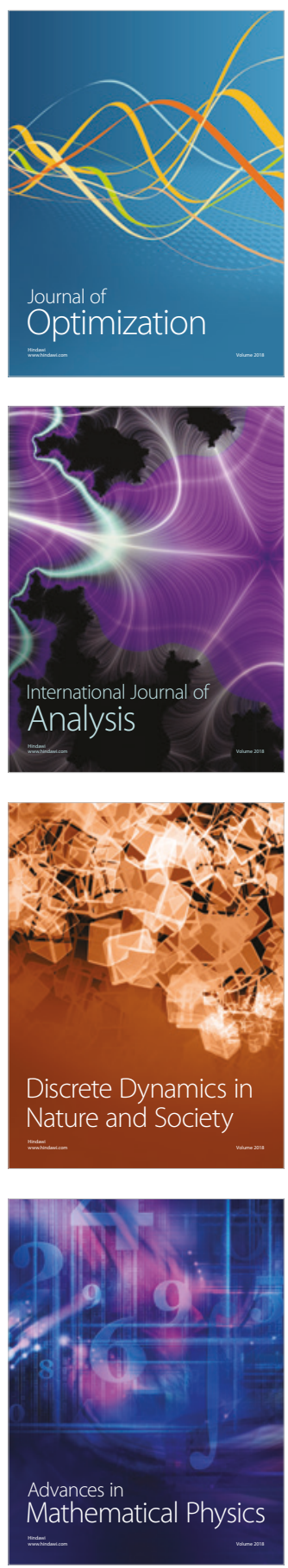